\title{
The GROUnd-based Secondary Eclipse project - GROUSE
}

\author{
Ernst de Mooij ${ }^{1}$, Remco de $\mathrm{Kok}^{2}$, Bas Nefs ${ }^{1}$, Matteo Brogi ${ }^{1}$ and \\ Ignas Snellen ${ }^{1}$ \\ ${ }^{1}$ Leiden Observatory, Leiden University, Postbus 9513, 2300 RA, Leiden, The Netherlands; \\ email: demooij@strw.leidenuniv.nl \\ ${ }^{2}$ SRON Netherlands Institute for Space Research, Sorbonnelaan 2, 3584 CA Utrecht, The \\ Netherlands;
}

\begin{abstract}
Secondary eclipse observations of exoplanets at near-infrared wavelengths are important to constrain the energy budgets of hot-Jupiters, since they probe the radiation from the planet's atmosphere at the peak of the spectral energy distribution. Since this wavelength range is accesible from the ground, we have started the GROUnd-based Secondary Eclipse (GROUSE) project. As part of the GROUSE project, we target a sample of hot-Jupiters at near-infrared and optical wavelengths. Planets include TrES-3b, HAT-P-1, WASP-18b and WASP-33b.
\end{abstract}

Keywords. techniques: photometric, planetary systems

\section{Introduction}

Secondary eclipse measurements of transiting extrasolar planets with the Spitzer Space Telescope have yielded many detections of thermal exoplanet light (e.g. Charbonneau et al. 2005; Deming et al. 2005; Knutson et al., 2008). One of the most interesting parts of the planet spectrum $(1-3 \mu \mathrm{m})$ is inaccessible with this satellite. Although the typical planet-to-star contrast ratio in this wavelength range is smaller than at the mid-infrared region probed by Spitzer, the near-infrared region is at the peak of the planet's spectral energy distribution and is also a wavelength range where molecular absorption bands can significantly influence the measured spectrum. Since there are several windows in the Earth's atmosphere in the near-infrared, it is possible to use ground-based telescopes to study the properties of the exoplanet atmospheres in this range, which, during the past few years, has led to the detection of the secondary eclipses of several exoplanets (e.g. De Mooij \& Snellen 2009; Sing \& López-Morales 2009; Croll et al. 2010).

\section{The GROUSE project}

We have started the GROUnd-based Secondary-Eclipse (GROUSE) project to study the optical and near-infrared spectral energy distributions of a sample of hot-Jupiters. For this we use a variety of instruments and telescopes, including LIRIS at the William Herschel Telescope for near-infrared observations, and OSIRIS on the Gran Telescopio Canarias and the WFC on the Isaac Newton Telescope for our optical measurements.

The sample we target can be divided into two parts, the first part contains the hottest and brightest exoplanet systems, such as HAT-P-7b and WASP-18b, for which we should be able to reach a sufficient signal-to-noise ratio to detect their secondary eclipses at both optical and near-infrared wavelengths. The second part of the sample contains planets for which we only expect to be able to measure the the planet's light in K-band, such as HAT-P-1b. 

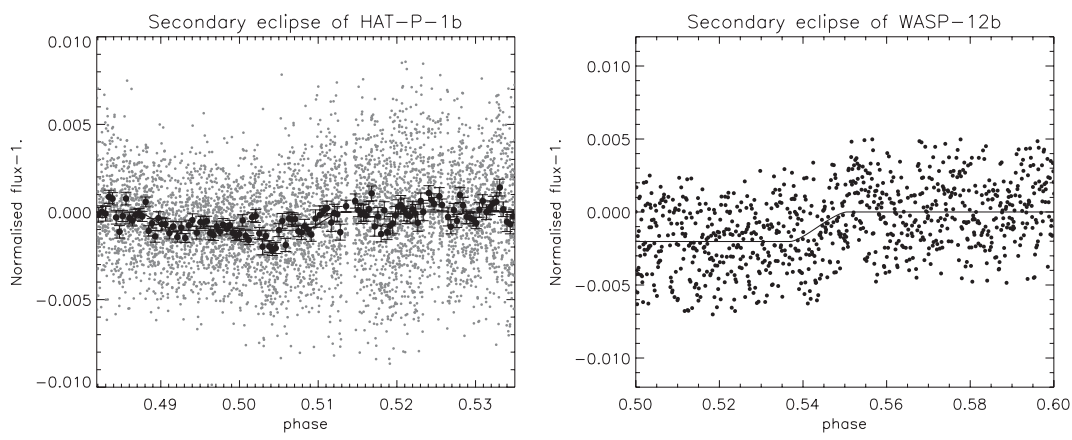

Figure 1. Two examples of secondary eclipse observations from the GROUSE project. Left panel: The Ks-band secondary eclipse of HAT-P-1b (De Mooij et al. 2011), the black points show the data binned by 41 frames. Right panel: Partial Ks-band secondary eclipse of WASP-12b observed with the William Herschel Telescope.

As part of this project we have, so far, reported on the detections in Ks-band of the secondary eclipses of TrES-3b (De Mooij \& Snellen 2009; Paper I) and HAT-P-1b (De Mooij et al. 2011; Paper II). Two examples of secondary eclipse observations are shown in Fig. 1.

Since secondary eclipse observations require very high signal-to-noise ratios, we use a specialised strategy to reduce systematic effects, and to minimize the overheads. Since the best candidate systems are relatively bright $(\mathrm{V} \lesssim 12)$, we defocus the telescope for all our observations. The defocusing has the additional benefit of reducing the sensitivity of the observations to flatfielding errors, but at the cost of an increased noise due to the sky background.

Contrary to typical near-infrared observations, where a dither sequence is used to determine the sky background, we perform our observations in staring mode, which enables us to use guiding without increasing the overheads due to the reacquisition of the guide star. This is very important for the stability of the observations, since guiding enables us to maintain the position of the star within a few pixels for the entire duration of the observation. For the background subtraction, we acquire a set of images on a blank field before and/or after the eclipse.

Since the eclipse depth has to be measured with respect to the out-of-eclipse baseline, we aim to observe the target out-of eclipse for as long as possible. Typically we try to get a baseline that is longer than the eclipse duration, since systematic effects on timescales of the eclipse will then be visible. In this way we manage to obtain eclipse depth uncertainties down to $\sim 0.2$ millimag in K-band. This allows us to put tight constraints on the planets' energy budgets, which is especially important now that Spitzer has entered its warm mission.

\section{References}

Charbonneau, D., et al. 2005, ApJ, 626, 523

Croll, B., Jayawardhana, R., Fortney, J. J., Lafrenire, D., \& Albert, L. 2010, ApJ, 718, 920

Deming, D., Seager, S., Richardson, L. J., \& Harrington, J. 2005, Nature, 434, 740

De Mooij, E. J. W. \& Snellen, I. A. G. 2009, A\&A, 493, L35

De Mooij, E. J. W., De Kok, R. J., Nefs, S. V., \& Snellen, I. A. G. 2011, A\&\&A, 528, A49

Knutson, H. A., et al. 2008, ApJ, 673, 526

Sing, D. K. \& López-Morales, M. 2009, A\& A, 493, L31 